\section{Ingestão de folato nos períodos pré e pós- fortificação mandatória: estudo de base populacional em São Paulo, Brasil}

\author{
Folic acid intake before and after mandatory \\ fortification: a population-based study in \\ São Paulo, Brazil
}

\author{
Ingesta de folatos en la prefortificación o \\ posfortificación: estudio de base poblacional \\ en São Paulo, Brasil
}

\begin{abstract}
This study analyzed folic acid intake before and since mandatory fortification. Dietary data were collected by 24-hour recall in a health survey in São Paulo (ISA-Capital) in 2003 and 2007-2008, stratifying the population according to life stage and gender. Estimated average requirement (EAR) and tolerable upper intake level (UL) were used to assess intake. Prevalence of inadequate folic acid intake decreased in all groups, especially adolescents and adult males (72\% to $<1 \%$ and $76 \%$ to $6 \%$, respectively) but remained high in adult women (38\%). Beans were the main source of folic acid before fortification. With fortification, bread became the main source, but beans remained important. Fortification was successful (increased intake within safe levels), but it raised concerns about the high proportion in the target group (adult women) who still fail to meet the recommended intake.
\end{abstract}

Folic Acid; Fortified Food; Food Consumption
Dirce Maria Lobo Marchioni 1

Eliseu Verly-Jr. ${ }^{2}$

Josiane Steluti 1

Chester Luis Galvão Cesar 1

Regina Mara Fisberg 1

\section{Resumo}

Analisou-se a ingestão de folato nos periodos pré e pós-fortificação. Os dados dietéticos foram coletados por recordatório de 24 horas (R24h) no Inquérito de Saúde de São Paulo (ISA-Capital) em 2003 e 2007/2008, estratificando-se a população segundo fase da vida e sexo. Utilizaram-se os valores de recomendação: necessidade média estimada (EAR) e nível máximo de ingestão tolerada (UL) e o método "EAR como ponto de corte" para estimar a inadequação da ingestão. Houve redução na prevalência de inadequação da ingestão de folato em todos os estratos, com destaque para os adolescentes e adultos do sexo masculino, de $72 \%$ para < 1\% e de $76 \%$ para $6 \%$, mas nas mulheres adultas a inadequação permaneceu elevada (38\%). Antes da fortificação, o feijão foi o alimento que mais contribuiu para a ingestão de folato; após, o maior contribuinte passou a ser o pão, porém o feijão permaneceu importante. A fortificação foi bem-sucedida (aumentou a ingestão dentro de níveis seguros), porém, gera preocupação a elevada proporção no grupo alvo, mulheres adultas, que não atingem a recomendação para ingestão de folato.

Ácido Fólico; Alimentos Fortificados; Consumo de Alimentos 


\section{Introdução}

A fortificação mandatória de farinhas de trigo e milho com ácido fólico ocorre no Brasil desde 2004 1. Como nos Estados Unidos e Canadá, a fortificação no Brasil foi motivada pela expectativa de reduzir a incidência de má-formação de tubo neural em recém-nascidos, tendo em vista que uma importante forma de prevenção é a ingestão adequada de folato ${ }^{2}$. Apesar da escassez de dados nacionais representativos sobre a ingestão de folato, pesquisas em outros países já denunciavam o consumo insuficiente deste nutriente 3 . Nos países onde ocorreu a fortificação de alimentos foram observadas reduções na ocorrência de malformações do tubo neural 4,5,6,7,8,9, aumento do folato sérico e eritrocitário na população em geral e nas mulheres em idade fértil 10,11,12,13, tornando a fortificação mandatória com ácido fólico uma das mais bem-sucedidas ações de saúde pública para a redução dos defeitos de tubo neural ${ }^{8}$. No Brasil, foi encontrada uma redução em $39 \%$ da prevalência de espinha bífida, relacionada ao programa de fortificação mandatória de farinhas com ácido fólico 14 .

O folato tem importante papel na saúde 15 , estabilidade e manutenção do material genético 16 e, além do estabelecido papel na redução dos defeitos de tubo neural, estudos observacionais apontam para um efeito benéfico na proteção de alguns tipos de cânceres 17 e prevenção de acidente cerebral vascular 18 com o aumento da ingestão de folato. Porém, os efeitos do consumo excessivo de folato têm causado apreensão, devido ao seu potencial efeito de estímulo à proliferação de células cancerígenas 19 . A evidência epidemiológica sugere que o folato confere uma proteção contra o risco de câncer colorretal 20,21. Contudo, a relação é complexa, e os resultados de ensaios clínicos e estudos em animais sugerem que doses elevadas de ácido fólico podem aumentar o risco desse tipo de câncer em certas circunstâncias. Os mecanismos moleculares subjacentes ao efeito dual do folato na carcinogênese colorretal não são completamente compreendidos 22. Nos Estados Unidos e Canadá foi observada uma inversão na tendência, até então de queda, na incidência de câncer colorretal, que tem sido relacionada com o início da fortificação mandatória nestes países 23,24,25. Taxas absolutas de câncer de cólon e reto começaram a crescer a partir de 1996, nos Estados Unidos, e 1998, no Canadá.

No Brasil, passados nove anos do início da fortificação, pouco se sabe sobre o impacto desta política pública na ingestão dietética de folato. Pesquisa em um grupo de adolescentes, no período anterior à fortificação mandatória, reve- lou alta prevalência de inadequação da ingestão de folato; aproximadamente $89 \%$ da população estudada 26. Diante da hipótese do aumento do risco à saúde associado ao elevado consumo de ácido fólico, a avaliação da ingestão pós-fortificação deve ter como foco não somente a proporção da população com ingestão insuficiente, mas também grupos com nível de ingestão potencialmente insegura para a saúde. Medidas bioquímicas são preferidas como indicadores do impacto da fortificação, contudo, dados de consumo alimentar, com custos mais modestos se comparados aos biomarcadores, podem servir como alerta para a baixa ou excessiva ingestão desse nutriente.

O objetivo deste estudo foi investigar a ingestão de folato nos períodos pré e pós-fortificação, e verificar a contribuição de grupos de alimentos para a ingestão total de folato nos dois períodos estudados em uma amostra representativa da população.

\section{Metodologia \\ População de estudo}

O consumo alimentar foi avaliado no período préfortificação, durante o ano de 2003, e no período pós-fortificação, nos anos de 2007 e 2008. Foram utilizados os dados do Inquérito de Saúde de São Paulo (ISA-Capital 2003), estudo transversal de base populacional com amostra probabilística de residentes na área urbana do Município de São Paulo, Brasil, conduzido de março a dezembro de 2003. Detalhes do processo de amostragem do ISA-Capital são obtidos em Castro et al. ${ }^{27}$. Resumidamente, o processo de amostragem foi realizado em dois estágios: setores censitários e os domicílios. Para o sorteio, agruparam-se os setores em três estratos com base nos percentuais de chefes de família com nível universitário: menos de $5 \%$, $5 \%$ a $24,9 \%$ e com $25 \%$ e mais. Foram entrevistados 2.298 indivíduos, sendo 803 adolescentes (12 a 19 anos), 713 adultos (20 a 59 anos) e 782 idosos (60 anos ou mais), de ambos os sexos. Participantes do ISA-Capital 2003 foram contatados para responder a um novo inquérito alimentar entre 2007 e 2008. Foram localizados e concordaram em participar 861 indivíduos, sendo 214 adolescentes, 357 adultos e 290 idosos. Para comparar essa amostra com a inicial, verificou-se se a perda era diferencial em relação aos setores, o que poderia demostrar que setores em bairros mais abastados deixariam de ser representados. Observou-se que foram contemplados 60 setores censitários dos 61 utilizados na amostra inicial. Também não foram observadas dife- 
renças entre as amostras de 2003 e 2007 quanto à escolaridade do chefe da família. A escolaridade do chefe da família foi selecionada em vez da escolaridade do indivíduo, pois nos adolescentes esta variável é dependente da idade e enviesaria a comparação ente as faixas etárias, e por representar nível socioeconômico.

O projeto foi submetido e aprovado pelo Comitê de Ética em Pesquisa da Faculdade de Saúde Pública, Universidade de São Paulo. Todos os participantes assinaram o Termo de Consentimento Livre e Esclarecido.

\section{Coleta e processamento dos dados}

As entrevistas foram conduzidas nos domicílios por entrevistadores previamente treinados. O consumo alimentar foi coletado pelo método Recordatório de 24 horas (R24h), utilizando o procedimento recomendado por Thompson \& Byers 28 . A coleta dos dados de consumo dos períodos pré e pós-fortificação foi realizada aleatoriamente entre os dias da semana e meses do ano. Anteriormente à digitação dos dados de consumo alimentar, as informações contidas em cada uma das coletas foram checadas a fim de monitorar a qualidade das entrevistas e definir a padronização para quantidades dos alimentos e receitas das preparações relatadas. O programa Nutrition Data System for Research (NDSR) versão 2007 (Nutrition Coordinating Center, University of Minnesota, Minneapolis, Estados Unidos), que tem como principal base de dados a tabela norte-americana do Departamento de Agricultura dos Estados Unidos (USDA), foi utilizado para estimar as formas de folato encontradas nos alimentos e calcular os equivalentes dietéticos de folato (dietary folate equivalent - DFE) 15,29. Assim, foram obtidos os valores de ingestão do folato que ocorre naturalmente nos alimentos (folato natural), do acido fólico, a forma sintética da vitamina usada na fortificação e em suplementos, e de equivalentes dietéticos de folato, que consideram as diferenças na absorção das formas sintética e natural do folato. Os valores de folato sintético dos alimentos e preparações, consequentemente os valores de DFE, foram corrigidos considerando a diferença da quantidade adicionada de ácido fólico nas farinhas do Brasil (150mcg por 100g) e dos Estados Unidos (140mcg por 100g).

Uma questão adicional foi realizada para verificar o uso de suplementos dietéticos. Contudo, essa informação não foi utilizada na totalização da ingestão de ácido fólico pela baixa prevalência de uso de suplementos, cerca de 4,5\%.
Estimativa da ingestão habitual de folato nos períodos pré e pós-fortificação

Dados de consumo baseados em um ou poucos R24h apresentam a distribuição inflada em virtude da variabilidade do dia a dia do consumo, ou seja, a variabilidade intrapessoal. Consequentemente, percentis de consumo, bem como a proporção de indivíduos acima de um dado ponto de corte são sub ou superestimados. A ingestão habitual, entretanto, pode ser estimada utilizando-se métodos estatísticos que removem a variabilidade intrapessoal. Esses requerem a aplicação de um segundo R24h em pelo menos uma subamostra da população de estudo ${ }^{30}$. Se apenas um R24h tiver sido coletado, é recomendada a utilização de variância intrapessoal externa, obtida preferencialmente de pesquisas com população semelhante 31 .

Em 2003, período pré-fortificação, entretanto foi coletado somente um único R24h para cada indivíduo. Em 2007, aplicaram-se dois R24h, sendo que o primeiro deles foi realizado no domicílio e o segundo por telefone. O intervalo entre o primeiro e o segundo R24h para cada indivíduo foi de aproximadamente dois meses. Dessa maneira, a variância intrapessoal calculada com base nessa repetição foi então utilizada na estimativa da distribuição da ingestão habitual: (a) variância intrapessoal externa da ingestão de folato natural para estimar a ingestão de folato no período pré-fortificação; e (b) variância intrapessoal da ingestão de DFE para estimar a ingestão de folato no período pós-fortificação. Para o cálculo da variância intrapessoal e estimativa da ingestão habitual foi utilizado o método proposto pela Iowa State University 32 .

\section{Comparação com as recomendações de ingestão}

Como parâmetros para avaliação da ingestão de folato foram utilizados os valores de recomendação propostos pelo Institute of Medicine (IOM), referentes à necessidade média estimada (estimated average requirement - EAR) e ao nível máximo de ingestão tolerada (upper tolarable intake level - UL) que é expressa em $\mu$ g de ácido fólico/ dia ${ }^{15}$. A prevalência de inadequação da ingestão de folato foi calculada utilizando-se o método da EAR como ponto de corte 15,33 , e foi calculado o percentual de indivíduos com consumo acima da UL. A população foi estratificada segundo fase da vida (adolescentes, adultos e idosos) e sexo, conforme categorias para os valores de recomendação de consumo. 


\section{Contribuição para ingestão de folato}

Para cada faixa etária, foram listados todos os alimentos que contribuíram para ingestão de folato, cujo somatório dos percentuais cobrisse até $80 \%$ de todo folato proveniente de todos os alimentos relatados nos R24h. Posteriormente, os alimentos foram agrupados em cinco categorias: pães, massas (macarrão, lasanha, nhoque etc.), salgados fritos e assados (esfirra, coxinha, pastel frito, empada, bolinho de queijo, rissole, enroladinho, pastel assado e quibe), bolos e biscoitos (doces, salgados, recheados), feijões e frutas, legumes e verduras. O percentual de contribuição de cada grupo correspondeu à soma dos percentuais de contribuição de cada alimento.

As prevalências de inadequação e os percentuais de contribuição dos alimentos para ingestão de folato foram calculados considerando-se a expansão e o desenho complexo da amostra. Intervalos de confiança foram calculados com base em erros-padrão obtidos pela técnica de replicação Jackknife.

\section{Resultados}

A ingestão habitual estimada (média e desvio-padrão) de folato e a proporção de indivíduos que não atingem a recomendação, nos períodos pré e pós-fortificação, são mostradas na Tabela 1. Para todos os subgrupos estudados houve aumento da média de ingestão e consequentemente importante redução da prevalência de inadequação da ingestão de folato. Entre os adolescentes, a redução da inadequação foi de $90 \%$ nas mulheres e virtualmente $100 \%$ nos homens. Em adultos, a redução para os homens foi de $92 \%$ contra $60 \%$ para as mulheres. Mesmo com a redução, as mulheres ainda permanecem com elevada inadequação da ingestão (38\%). Entre os idosos, a redução foi de $77 \%$ para os homens e $75 \%$ para as mulheres.

As médias de ingestão de folato, computadas em DFE, elevaram em todos os grupos etários, variando de $158 \mu \mathrm{g}$ a $285 \mu \mathrm{g}$ no período pré-for-

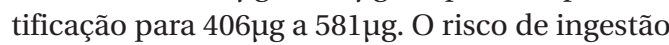
excessiva de ácido fólico, ou seja, a ingestão habitual da forma sintética da vitamina acima do valor da UL (800mcg para adolescentes e $1.000 \mathrm{mcg}$ para adultos e idosos) foi menor que $1 \%$ em todos os estratos de sexo e idade analisados.

A Figura 1 mostra a contribuição de grupos de alimentos para a ingestão de folato. Antes da fortificação, o alimento que mais contribuiu para a ingestão de folato foi o feijão, seguido por frutas, legumes e verduras e pães. O grupo frutas, legumes e verduras contribuiu com quase $30 \%$ da ingestão total de folato entre os idosos, ao passo que entre os adolescentes a contribuição foi de $13 \%$. Após a fortificação, a participação relativa dos alimentos à base de farinha aumentou, co-

Necessidade média estimada (EAR), ingestão de folato (média, desvio-padrão - DP, intervalo de 95\% de confiança - IC95\%) e prevalência de inadequação da ingestão de folato * nos períodos pré e pós-fortificação, segundo sexo e faixa etária. São Paulo, Brasil.

\begin{tabular}{|c|c|c|c|c|c|c|c|c|c|}
\hline & \multirow[t]{3}{*}{ EAR } & \multicolumn{5}{|c|}{ Período pré-fortificação } & \multicolumn{3}{|c|}{ Período pós-fortificação } \\
\hline & & \multirow[t]{2}{*}{$\mathrm{n}$} & \multicolumn{2}{|c|}{$\begin{array}{c}\text { Ingestão de } \\
\text { folato ( } \mu \text { g DFE) }\end{array}$} & \multirow{2}{*}{$\begin{array}{l}\text { Prevalência de } \\
\text { Inadequação } \\
\text { de folato } \\
\% \text { (IC95\%) }\end{array}$} & \multirow[t]{2}{*}{$\mathrm{n}$} & \multicolumn{2}{|c|}{$\begin{array}{c}\text { Ingestão de } \\
\text { folato ( } \mu \text { g DFE) }\end{array}$} & \multirow{2}{*}{$\begin{array}{c}\text { Prevalência de } \\
\text { Inadequação } \\
\text { de folato } \\
\text { \% (IC95\%) }\end{array}$} \\
\hline & & & Média (DP) & IC95\% & & & Média (DP) & IC95\% & \\
\hline \multicolumn{10}{|l|}{ Masculino } \\
\hline Adolescentes & 330 & 271 & $236,8(66,5)$ & $227,7-245,8$ & $72(62-81)$ & 108 & $580,9(142,3)$ & $504,6-657,1$ & $<1 \star \star$ \\
\hline Adultos & 320 & 385 & $284,7(66,3)$ & $267,8-301,5$ & $76(67-84)$ & 169 & $567,9(170,7)$ & $505,1-630,6$ & $6(0-15)$ \\
\hline Idosos & 320 & 388 & $157,2(65,9)$ & $144,6-169,7$ & $83(77-88)$ & 134 & $498,7(149,0)$ & $403,4-593,9$ & 19 (8-29) \\
\hline \multicolumn{10}{|l|}{ Feminino } \\
\hline Adolescentes & 330 & 240 & $219,2(84,8)$ & $193,4-244,9$ & $88(80-94)$ & 106 & $452,0(146,1)$ & $383,2-520,7$ & $9(0-34)$ \\
\hline Adultos & 320 & 434 & $198,2(63,2)$ & $184,6-211,7$ & $95(92-97)$ & 188 & $409,7(198,0)$ & $369,3-450,0$ & $38(23-51)$ \\
\hline Idosos & 320 & 408 & $198,9(60,0)$ & $184,7-213,0$ & $96(93-98)$ & 156 & $406,0(103,6)$ & $372,2-439,7$ & 24 (8-39) \\
\hline
\end{tabular}

DFE: equivalentes dietéticos de folato.

* Percentual abaixo da EAR;

** Erro padrão não calculado para prevalência menor que $1 \%$. 
Grupos de alimentos segundo percentuais de contribuição para a ingestão de folato e respectivos intervalos de 95\% de confiança (IC95\%) nos períodos pré e pós-fortificação de alimentos com ácido fólico. São Paulo, Brasil.
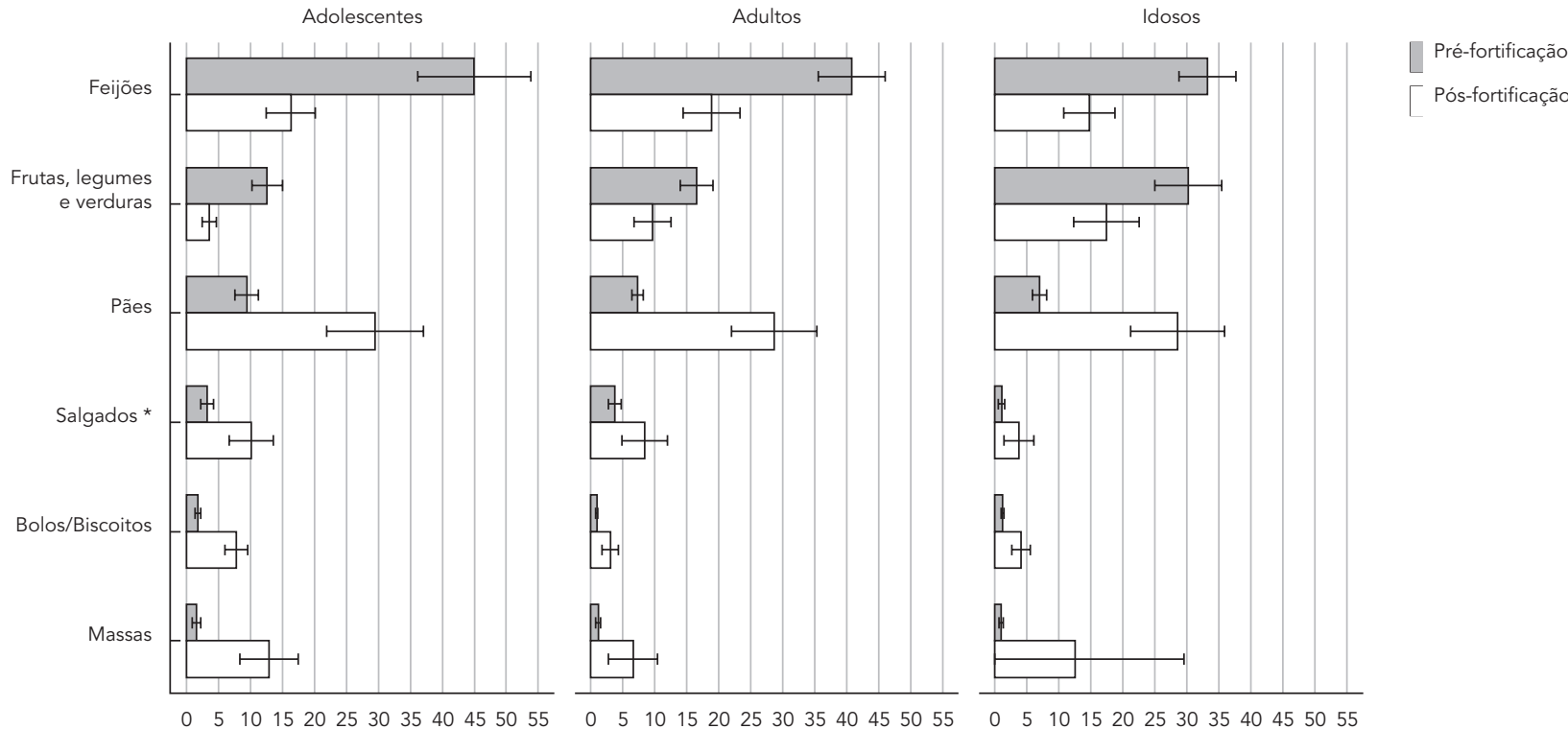

Contribuição (\%) para ingestão de folato

* Salgados: esfirra, coxinha, pastel frito, empada, bolinho de queijo, rissole, enroladinho, pastel assado e quibe.

mo esperado, e a participação do feijão, frutas, legumes e verduras diminuiu em todos os grupos etários. Essa mudança, porém, foi mais pronunciada entre os adolescentes, cuja contribuição do feijão passou de $45 \%$ para $16 \%$ e de frutas, legumes e verduras de $13 \%$ para $4 \%$, ao mesmo tempo em que a contribuição dos salgados, biscoitos, massas e pães juntos elevou de $16 \%$ para $60 \%$.

\section{Discussão}

O presente estudo avaliou a ingestão de folato na cidade de São Paulo nos períodos pré e pós- fortificação mandatória de farinhas de trigo e milho com ácido fólico e observou, em toda a população: (1) importante redução da prevalência de inadequação da ingestão de folato; (2) alterações nos grupos alimentares que contribuíram para a ingestão de folato; e (3) praticamente inexistente proporção de indivíduos com ingestão excessiva.

Dos principais achados, destaca-se um declínio importante, entre $60 \%$ a quase $100 \%$, na prevalência de inadequação de ingestão de folato, considerada como o percentual da população que não atinge o valor de recomendação correspondente a EAR, em todas as faixas etárias e em ambos os sexos, similar a outros países que adotaram a fortificação com ácido fólico de caráter mandatório 13,34,35,36. Em relação aos grupos estudados, nota-se que os adolescentes apresentaram as maiores reduções, possivelmente em decorrência do consumo de alimentos fortificados.

Entre os adolescentes de ambos os sexos e adultos do sexo masculino foram observadas prevalências baixas $(<10 \%)$. Esses valores foram menores que os estimados para a população brasileira com base nos dados de consumo alimentar individual coletados no Inquérito Nacional de Alimentação da Pesquisa de Orçamentos Familiares 2008-2009 (INA/POF 2008-2009) 37, e semelhantes aos observados no Canadá 35 e nos Estados Unidos 34 . Porém, no grupo dos idosos e mulheres adultas foram observadas prevalências maiores que nesses países 34,35. Chama a atenção que quase $40 \%$ das mulheres adultas não apresentaram ingestão habitual nos valores recomendados de folato, tendo em vista que este grupo é o principal alvo da política pública de fortificação. 
O grupo dos pães destacou-se como o maior contribuinte para a ingestão de folato, independentemente da faixa etária, o que pode ser explicado pela participação expressiva destes alimentos na dieta $(63,6 \%$ consumiram estes alimentos no dia avaliado da dieta) segundo os dados publicados no INA/POF 2008-2009 37. A presença do grupo dos feijões entre os principais contribuintes para a ingestão da vitamina mesmo após a fortificação mandatória das farinhas é um achado relevante. As leguminosas, grupo do qual o feijão faz parte, tem atraído a atenção pois é consenso que o consumo de grãos contribui para uma dieta equilibrada e está associado à prevenção do risco de doenças crônicas, como as cardiovasculares e determinados cânceres 38,39. Porém, análises temporais, baseando-se nos dados da POF entre 1974 e 2003, mostram um declínio no consumo de alimentos básicos como arroz e feijão 40 .

Os resultados do presente trabalho apontam para a distinta participação dos grupos de alimentos como contribuintes para a ingestão de folato entre os grupos etários, com maior participação dos salgados, massas e biscoitos entre os adolescentes quando comparados com os adultos e idosos. Inegavelmente, a política de fortificação de farinhas com ácido fólico no Brasil pode ser considerada bem-sucedida, no entanto, há que se atentar para a participação de alimentos cujo consumo tem sido desestimulado nos guias alimentares nacionais, como os biscoitos e salgados 41 . Depreende-se que a continuidade dos esforços para a manutenção do hábito de consumo de arroz e feijão e a elevação do consumo de frutas, legumes e verduras pela população, influenciará de forma positiva a ingestão de folato natural.

Nos Estados Unidos e Canadá, pioneiros na adoção dessa política, observou-se uma evolução positiva dos parâmetros nutricionais de folato, queda da prevalência de inadequação 34,42 e aumento dos níveis sanguíneos 10,43. As recomendações para ingestão de folato foram baseadas na manutenção da concentração de folato eritrocitário, considerado indicador primário da inadequação devido à sua correlação com o folato armazenado no fígado, enquanto os UL foram determinados em virtude de evidências limitadas, porém sugestivas, de que o excesso de ácido fólico possa precipitar ou exacerbar neuropatias em indivíduos com deficiência de B12. Enfatizase que o consumo de suplementos motivou o estabelecimento do valor limítrofe, acima do qual há risco de ocorrência de efeitos danosos 15 . Porquanto, é necessário acompanhar os efeitos da fortificação dos alimentos. No presente trabalho, não foi observada expressiva ingestão de folato acima da UL no período após a fortificação, ao contrário dos Estados Unidos, onde, entre 2001 e $2008,2,5 \%$ da população adulta excederam este limite 44

Ao mesmo tempo em que o folato tem sido associado à diminuição de risco para determinadas doenças crônicas 45,46, há evidências que o excesso de folato, ao aumentar a síntese de DNA e estimular a proliferação celular, pode promover a progressão de lesão pré-maligna ou maligna preexistente 23,24 . Adicionalmente, tem sido constatada a presença de ácido fólico não metabolizado no sangue, decorrente da ingestão da forma sintética da vitamina 47,48,49. Apesar do desconhecimento sobre os efeitos biológicos do ácido fólico não metabolizado, este pode ser um fator relevante nas questões de segurança associadas com a alta ingestão de ácido fólico 47,50.

Outro ponto importante na discussão das recomendações de folato diz respeito à variabilidade genética das populações. A evolução da ciência, em especial do campo da genética e biologia molecular, permitiu avançar no conhecimento das diferenças entre as populações. Diversos polimorfismos de genes que codificam enzimas envolvidas no metabolismo do folato já foram identificados e, dentre os mais comuns, destaca-se a variante C677T da 5,10-metilenotetahidrofolato redutase (MTHFR) 51. Nos Estados Unidos, em uma amostra nacional representativa, encontrou-se uma frequência de $11 \%$ de homozigotos TT para a MTHFR C677T na população ${ }^{52}$. No Brasil, estudos registraram uma frequência do homozigoto TT entre 9,5-10\% 53,54. Sabe-se que a presença do polimorfismo C677T no gene da MTHFR está associada à diminuição da atividade da enzima, elevação sanguínea de homocisteína e ao risco aumentado da ocorrência de agravos cardiovasculares, neoplasias e outras doenças, e que o estado nutricional adequado da vitamina parece modular esta associação 55 . Achados como esses reforçam a importância de uma ingestão adequada às necessidades individuais.

Os resultados deste trabalho devem ser interpretados à luz de algumas considerações metodológicas. O R24h foi utilizado para a coleta de dados dietéticos e, apesar deste método ser considerado superior ao questionário de frequência alimentar, por ser de curto prazo, para espelhar a distribuição do consumo habitual na população é necessário que pelo menos duas aplicações sejam realizadas, seguidas de ajuste estatístico dos dados para consideração da variabilidade intrapessoal. Esses procedimentos foram seguidos neste estudo, e a obtenção da ingestão habitual do folato nos dois períodos pode ser considerada um dos pontos fortes desta pesquisa 56 . No entanto, na estimativa da ingestão de folato no 
período pré-fortificação foi assumida a mesma variância intrapessoal da ingestão observada no período posterior. Porém, foram utilizadas estimativas de variância intrapessoal geradas da replicação da medida dietética nos mesmos participantes do inquérito realizado em 2003, com um intervalo de quatro anos entre as coletas. Apesar desse intervalo, argumenta-se que o país vivenciou uma estabilidade econômica ao longo desse período, não ocorrendo eventos que pudessem caracterizar mudanças expressivas nos padrões de alimentação.

Outro ponto refere-se à perda de seguimento, que foi elevada. Não há dados publicados na literatura nacional para apreciar a perda de seguimento em estudos realizados em metrópoles das dimensões de São Paulo, e para este fim utilizamos a abordagem tradicional em epidemiologia, que foi comparar os indivíduos que permaneceram na coorte com os que participaram do inquérito de 2003. Apesar de não terem sido encontradas grandes diferenças, não se pode descartar que a representatividade da amostra tenha sido afetada. Ainda assim, é o primeiro estudo que apresenta dados antes e depois da fortificação, e por isto traz informações relevantes para a avaliação dessa política pública. Porém, novos estudos, preferencialmente com a inclusão de biomarcadores do estado nutricional de folato, devem ser realizados.

A obrigatoriedade da fortificação de farinhas de trigo e milho com ácido fólico reduziu a prevalência de inadequação da ingestão de folato, no entanto, alterou os alimentos contribuintes da vitamina. É necessário acompanhar e avaliar periodicamente as estratégias da política pública de fortificação mandatória, considerando: o consumo alimentar, sobretudo o risco potencial dos grupos populacionais que consomem altas quantidades de produtos à base de farinhas; o controle do conteúdo de ácido fólico adicionado às farinhas; as concentrações de folato sérico e eritrocitário; a prevalência de defeitos do tubo neural e outros defeitos de nascimento; e os resultados de estudos relacionados ao efeito adverso como câncer e outras doenças crônicas.

\section{Resumen}

Se analizó la ingesta de folato en los periodos de pre- $y$ posfortificación. Los datos dietéticos se recogieron mediante un recordatorio de 24 horas en la Encuesta de Salud de São Paulo (ISA-Capital) en 2003 y 2007/2008, estratificando la población según etapa de la vida y sexo. Se utilizaron los valores de recomendación: necesidad media estimada (EAR) y la ingesta máxima tolerable (UL) y el método "EAR como punto de corte" para estimar la ingesta inadecuada. Se observó una reducción en la prevalencia de ingesta inadecuada de folato en todos los grupos, especialmente en los adolescentes y adultos varones, de un $72 \%$ a $<1 \%$ y de un $76 \%$ a un $6 \%$, no obstante, en las mujeres adultas, la inadecuación se- guía siendo alta (38\%). Antes de la fortificación, los frijoles son el alimento que más contribuye a la ingesta de folato, después es el pan el que también constituye una fuente de folato, pese a que los frijoles siguen siendo los más importantes. La fortificación fue exitosa (aumento de la ingesta dentro de niveles seguros), sin embargo, se plantea una preocupación por el alto porcentaje del grupo objetivo: mujeres adultas que no cumplen con la recomendación para la ingesta de ácido fólico.

Ácido Fólico; Alimentos Fortificados; Consumo de Alimentos 


\section{Colaboradores}

D. M. L. Marchioni foi responsável pelo delineamento do estudo, orientou as análises e participou da redação e revisão do manuscrito. E. Verly-Jr. realizou as análises, participou da redação e revisão do manuscrito. J. Steluti participou da redação e revisão do manuscrito. C. L. G. César foi responsável pelo delineamento do estudo e pela revisão crítica do manuscrito. R. M. Fisberg foi responsável pelo delineamento do estudo, participou da redação e revisão do manuscrito.

\section{Referências}

1. Ministério da Saúde. Resolução no 344, de 13 de dezembro de 2002. Aprova o regulamento técnico para a fortificação das farinhas de trigo e das farinhas de milho com ferro ácido fólico, constante no anexo desta resolução. Diário Oficial da União 2002; 18 dez.

2. Laura E, Villarreal M, Arredondo P, Hernandez R, Jesus Z ,Villarreal JZ. Weekly administration of folic acid and epidemiology of neural tube defects. Matern Child Health J 2006; 10:397-401.

3. Quinlivan EP, Gregory 3rd JF. Effect of food fortification on folic acid intake in the United States. Am J Clin Nutr 2003; 77:221-5.

4. Honein MA, Paulozzi LJ, Mathews TJ, Erickson JD, Wong LY. Impact of folic acid fortification of the US food supply on the occurrence of neural tube defects. JAMA 2001; 285:2981-6.

5. Amarin ZO, Obeidat AZ. Effect of folic acid fortification on the incidence of neural tube defects. Paediatr Perinat Epidemiol 2010; 24:349-51.

6. Blencowe H, Cousens S, Modell B, Lawn J. Folic acid to reduce neonatal mortality from neural tube disorders. Int J Epidemiol 2010; 39 Suppl 1:i110-21.

7. Barboza Argüello ML, Umaña Solís LM. Impacto de la fortificación de alimentos con ácido fólico en los defectos del tubo neural en Costa Rica. Rev Panam Salud Pública 2011; 30:1-6.

\section{Agradecimentos}

Agradecemos a todos os pesquisadores, professores e alunos do Grupo de Pesquisa de Avaliação do Consumo Alimentar (GAC) pela dedicação e trabalho no desenvolvimento do inquérito de saúde ISA-Capital. À Secretaria Municipal da Saúde de São Paulo, FAPESP e CNPq pelo financiamento.
8. Berry RJ, Bailey L, Mulinare J, Bower C; Folic Acid Working Group. Fortification of flour with folic acid. Food Nutr Bull 2010; 31 Suppl 1:S22-35.

9. Lopez-Camelo JS, Castilla EE, Orioli IM; Instituto Nacional de Genética Médica Populacional; Estudio Colaborativo Latino Americano de Malformaciones Congénitas. Folic acid flour fortification: impact on the frequencies of 52 congenital anomaly types in three South American countries. Am J Med Genet 2010; 152A:2444-58.

10. Pfeiffer CM, Hughes JP, Lacher DA, Bailey RL, Berry RJ, Zhang M, et al. Estimation of trends in serum and RBC folate in the U.S. population from pre- to post fortification using assay-adjusted data from the NHANES 1988-2010. J Nutr 2012; 142:886-93.

11. Brown RD, Langshaw MR, Uhr EJ, Gibson JN, Joshua DE. The impact of mandatory fortification of flour with folic acid on the blood folate levels of an Australian population. Med J Aust 2011; 194:65-7.

12. Colapinto CK, O’Connor DL, Tremblay MS. Folate status of the population in the Canadian Health Measures Survey. CMAJ 2011; 183:E100-6.

13. Gall S, Seal J, Taylor R, Dwyer T, Venn A. Folate status and socio-demographic predictors of folate status, among a national cohort of women aged 26-36 in Australia, 2004-2006. Aust N Z J Public Health 2012; 36:421-6. 
14. Orioli IM, Nascimento RL, López-Camelo JS, Castilla EE. Effects of folic acid fortification on spina bifida prevalence in Brazil. Birth Defects Res A Clin Mol Teratol 2011; 91:831-5.

15. Institute of Medicine. Dietary reference intakes: for thiamin, riboflavin, niacin, vitamin B6, folate, vitamin B12, pantothenic acid, biotin and choline. Washington DC: National Academy Press; 1998.

16. Fenech M. Folate (vitamin B9) and vitamin B12 and their function in the maintenance of nuclear and mitochondrial genome integrity. Mutat Res 2012; 733:21-33.

17. World Research Cancer Fund; American Institute for Cancer Research. Food, nutrition, physical activity and the prevention of cancer: a global perspective. Washington DC: American Institute for Cancer Research; 2007.

18. Huo Y, Qin X, Wang J, Sun N, Zeng Q, Xu X, et al. Efficacy of folic acid supplementation in stroke prevention: new insight from a meta-analysis. Int J Clin Pract 2012; 66:544-5.

19. Miller JW, Ulrich CM. Folic acid and cancer-where are we today? Lancet 2013. [Epub ahead of print].

20. Giovannucci E. Epidemiologic studies of folate and colorectal neoplasia: a review. J Nutr 2002; 132 (8 Suppl):2350S-5S.

21. Lee JE, Willett WC, Fuchs CS, Smith-Warner SA, Wu $\mathrm{K}, \mathrm{Ma}$ J, et al. Folate intake and risk of colorectal cancer and adenoma: modification by time. Am J Clin Nutr 2011; 93:817-25.

22. Williams EA. Folate, colorectal cancer and the involvement of DNA methylation. Proc Nutr Soc 2012; 71:592-7.

23. Kim YI. Role of folate in colon cancer development and progression. J Nutr 2003; 133(11 Suppl 1):3731S-9S.

24. Kim YI. Folic acid supplementation and cancer risk: point. Cancer Epidemiol Biomarkers Prev 2008; 17:2220-5.

25. Mason JB, Dickstein A, Jacques PF, Haggarty P, Selhub J, Dallal G, et al. A temporal association between folic acid fortification and an increase in colorectal cancer rates may be illuminating important biological principles: a hypothesis. Cancer Epidemiol Biomarkers Prev 2007; 16:1325-9.

26. Vitolo MR, Canal Q, Campagnolo PD, Gama CM. Factors associated with risk of low folate intake among adolescents. J Pediatr (Rio J.) 2006; 82:121-6.

27. Castro MA, Barros RR, Bueno MB, César CL, Fisberg RM. Trans fatty acid intake among the population of the city of São Paulo, Brazil. Rev Saúde Pública 2009; 43:991-7.

28. Thompson FE, Byers T. Dietary assessment resource manual. J Nutr 1994; 124(11 Suppl):2245S317 .

29. Suitor CW, Bailey LB. Dietary folate equivalents: interpretation and application. J Am Diet Assoc 2000; 100:88-94.

30. Guenther PM, Philip SK, Carriquiry AL. Development of an approach for estimating usual nutrient intake distributions at the population level. J Nutr 1997; 127:1106-12.
31. Jahns L, Carriquiry A, Arab L, Mroz TA, Popkin BM. Within- and between-person variation in nutrient intakes of Russian and U.S. children differs by sex and age. J Nutr 2004; 134:3114-20.

32. Nusser SM, Carriquiry AL, Dodd KW, Fuller WA. A semiparametric transformation approach to estimating usual daily intake distributions. J Am Stat Assoc 1996; 91:1440-9.

33. Beaton GH. Approaches to analysis of dietary data: relationship between planned analyses and choice of methodology. Am J Clin Nutr 1994; 59 Suppl:253S-61S.

34. Bailey RL, Dodd KW, Gahche JJ, Dwyer JT, McDowell MA, Yetley EA, et al. Total folate and folic acid intake from foods and dietary supplements in the United States: 2003-2006. Am J Clin Nutr 2010; 91:231-7.

35. Shakur YA, Garriguet D, Corey P, O'Connor DL. Folic acid fortification above mandated levels results in a low prevalence of folate inadequacy among Canadians. Am J Clin Nutr 2010; 92:818-25.

36. Abdollahi Z, Elmadfa I, Djazayery A, Golalipour MJ, Sadighi J, Salehi F, et al. Efficacy of flour fortification with folic acid in women of childbearing age in Iran. Ann Nutr Metab 2011; 58:188-96.

37. Instituto Brasileiro de Geografia e Estatística. Pesquisa de orçamentos familiares 2008-2009: análise do consumo alimentar pessoal no Brasil. Rio de Janeiro: Instituto Brasileiro de Geografia e Estatística; 2011.

38. Bouchenak M, Lamri-Senhadji M. Nutritional quality of legumes, and their role in cardiometabolic risk prevention: a review. J Med Food 2013. [Epub ahead of print].

39. Papanikolaou Y, Fulgoni 3rd VL. Bean consumption is associated with greater nutrient intake, reduced systolic blood pressure, lower body weight, and a smaller waist circumference in adults: results from the National Health and Nutrition Examination Survey 1999-2002. J Am Coll Nutr 2008; 27:569-76.

40. Levy-Costa RB, Sichieri R, Pontes NS, Monteiro CA. Household food availability in Brazil: distribution and trends (1974-2003). Rev Saúde Pública 2005; 39:530-40.

41. Coordenação Geral da Política de Alimentação e Nutrição, Secretaria de Atenção à Saúde, Ministério da Saúde. Guia alimentar da população brasileira: promovendo a alimentação saudável. Brasília: Ministério da Saúde; 2005. (Série A. Normas e Manuais Técnicos).

42. Dietrich M, Brown CJ, Block G. The effect of folate fortification of cereal-grain products on blood folate status, dietary folate intake, and dietary folate sources among adult non-supplement users in the United States. J Am Coll Nutr 2005; 24:266-74.

43. Yang Q, Cogswell ME, Hamner HC, Carriquiry A, Bailey LB, Pfeiffer CM, et al. Folic acid source, usual intake, and folate and vitamin B-12 status in US adults: National Health and Nutrition Examination Survey (NHANES) 2003-2006. Am J Clin Nutr 2010; 91:64-72. 
44. Hamner HC, Tinker SC, Berry RJ, Mulinare J. Modeling fortification of corn masa flour with folic acid: the potential impact on exceeding the tolerable upper intake level for folic acid, NHANES 20012008. Food Nutr Res 2013. [Epub ahead of print].

45. Vollset SE, Clarke R, Lewington S, Ebbing M, Halsey J, Lonn E, et al. Effects of folic acid supplementation on overall and site-specific cancer incidence during the randomised trials: meta-analyses of data on 50,000 individuals. Lancet 2013. [Epub ahead of print].

46. Qin X, Cui Y, Shen L, Sun N, Zhang Y, Li J, et al. Folic acid supplementation and cancer risk: a metaanalysis of randomized controlled trials. Int J Cancer 2013. [Epub ahead of print].

47. Kalmbach RD, Choumenkovitch SF, Troen AM, D'Agostino R, Jacques PF, Selhub J. Circulating folic acid in plasma: relation to folic acid fortification. Am J Clin Nutr 2008; 88:763-8.

48. Morris MS, Jacques PF, Rosenberg IH, Selhub J. Circulating unmetabolized folic acid and 5-methyltetrahydrofolate in relation to anemia, macrocytosis, and cognitive test performance in American seniors. Am J Clin Nutr 2010; 91:1733-44.

49. Boilson A, Staines A, Kelleher CC, Daly L, Shirley I, Shrivastava A, et al. Unmetabolized folic acid prevalence is widespread in the older Irish population despite the lack of a mandatory fortification program. Am J Clin Nutr 2012; 96:613-21.

50. Troen AM, Mitchell B, Sorensen B, Wener MH, Johnston A, Wood B, et al. Unmetabolized folic acid in plasma is associated with reduced natural killer cell cytotoxicity among postmenopausal women. J Nutr 2006; 136:189-94.
51. Tanaka T, Scheet P, Giusti B, Bandinelli S, Piras MG, Usala G, et al. Genome-wide association study of vitamin B6, vitamin B12, folate, and homocysteine blood concentrations. Am J Hum Genet 2009; 84:477-82.

52. Yang QH, Botto LD, Gallagher M, Friedman JM, Sanders CL, Koontz D, et al. Prevalence and effects of gene-gene and gene-nutrient interactions on serum folate and serum total homocysteine concentrations in the United States: findings from the third National Health and Nutrition Examination Survey DNA Bank. Am J Clin Nutr 2008; 88:232-46.

53. Shinjo SK, Oba-Shinjo SM, Silva R, Barbosa KC, Yamamoto F, Scaff M, et al. Methylenetetrahydrofolate reductase gene polymorphism is not related to the risk of ischemic cerebrovascular disease in a Brazilian population. Clinics 2007; 63:295-300.

54. Ma E, Iwasaki M, Junko I, Hamada GS, Nishimoto IN, Carvalho SM, et al. Dietary intake of folate, vitamin B6, and vitamin B12, genetic polymorphism of related enzymes, and risk of breast cancer: a case-control study in Brazilian women. BMC Cancer 2009; 9:122.

55. Clifford AJ, Chen K, McWade L, Rincon G, Kim $\mathrm{SH}$, Holstege DM, et al. Gender and single nucleotide polymorphisms in MTHFR, BHMT, SPTLC1, CRBP2, CETP, and SCARB1 are significant predictors of plasma homocysteine normalized by RBC folate in healthy adults. J Nutr 2012; 142:1764-71.

56. Dodd KW, Guenther PM, Freedman LS, Subar AF, Kipnis V, Midthune D, et al. Statistical methods for estimating usual intake of nutrients and foods: a review of the theory. J Am Diet Assoc 2006; 106:1640-50.

Recebido em 14/Jun/2012

Versão final reapresentada em 05/Mai/2013

Aprovado em 08/Mai/2013 